$\angle$ Research Square

\title{
A Pilot Study on Developing Quantitative Indices for Age-Related Changes in Maxillary Primary Molar Root Canal Morphology
}

\section{Guili Dou}

Peking University School and Hospital of Stomatology

Bin Xia ( $\nabla$ summerinbeijing@vip.sina.com )

Peking University School and Hospital of Stomatology

Yijiao Zhao

Peking University School and Hospital of Stomatology

Zhipeng Sun

Peking University School and Hospital of Stomatology

\section{Research Article}

Keywords: Quantitative indices, Cone-beam computed tomography; Primary molar; Root canal morphology; Age

Posted Date: July 29th, 2021

DOI: https://doi.org/10.21203/rs.3.rs-745757/v1

License: (ㅇ) (i) This work is licensed under a Creative Commons Attribution 4.0 International License. Read Full License 


\section{Abstract}

Objectives To elucidate age-related changes in the root canal morphology of primary molars and determine new indices to detail the root canal anatomy by cone-beam computed tomography (CBCT).

Materials and Methods CBCT data of primary molars from 57 children aged 3-8 years without systemic diseases that affect tooth development were collected. The following indices were defined and calculated using Mimics 17.0, 3-Matic 9.0, and ImageJ software: (i) the cross-sectional area of the root canal; (ii) $R_{.15}$ : the proportion of the lateral canal wall with an inner diameter less than $0.15 \mathrm{~mm}$; (iii) $L_{C} / L_{R}$ : the ratio between the canal length and root length; and (iv) $\mathrm{N}_{\text {dis }}$ : the discontinuous canal count. Correlation analysis and binary logistic regression were performed to assess the variants and age-related changes in canal morphology.

Results On CBCT analysis, the cross-sectional area of the root canals for mesiobuccal (MB) roots, palatal $(\mathrm{P})$ roots and distobuccal $(\mathrm{DB})$ roots was negatively correlated with age $(P<0.05)$, with a correlation coefficient of $-0.41,-0.42$, and -0.26 , respectively. The $R_{.15}$ of MB roots was positively correlated with age $\left(P<0.05, r_{s}=0.39\right)$. The value of $L_{C} / L_{R}$ for $M B, D B$, and $P$ roots decreased with age, with a correlation coefficient of $-0.39,-0.42$, and -0.29 , respectively $(P<0.05)$. The risk of discontinuous canals $\left(\mathrm{N}_{\text {dis }}\right)$ for $\mathrm{MB}$, DB, and $\mathrm{P}$ roots increased with age $(P<0.05)$, with an odds ratio of $1.61,1.48$, and 1.60 respectively.

Conclusions The new indices developed in the study based on CBCT successfully demonstrate age-related changes in the root canals of primary molars.

\section{Introduction}

Primary root canal treatment (RCT) is a classic treatment that can preserve pulp infected teeth without pathological signs, which is beneficial for maintaining the integrity of dentition and masticatory function[1]. A thorough knowledge of the root canal anatomy of primary teeth is critical for successful RCT. According to previous researches on permanent teeth, canal morphology is genetically determined[2-4] but also changes with age[5]. The continuous formation of secondary dentin can cause structural changes in the root canal system [6]. One study on permanent teeth found that younger groups show a greater prevalence of a single root canal configuration (Vertucci type I) than older ones in the second maxillary premolars [7]. For mandibular second primary molars, the area of the root canal orifice of the mesial root decreased with age[8]. In addition, Amano et al. found that the volume ratio of pulp chambers to the crown in maxillary second deciduous molars in the mixed dentition stage was significantly smaller than that in the primary dentition stage[9]. Compared with the pulp chamber, the root canal system has more influence on RCT, but unfortunately, for decades, studies on the canal anatomy changes of primary teeth with age have been underexplored. Hibbard et al. found that there were more variations in root canal anatomy in primary molars with root resorption than in those without root resorption, but the age of children whose teeth were included in this study were unknown [10]. 
Various methods are applied in studies on canal anatomy. Canal staining and clearing is generally used to describe canal morphology[11, 12], but this approach destroys the tooth integrity. Micro-computed tomography (micro-CT) can be used to describe the three-dimensional (3D) shape of the root canal in detail with high resolution and is non-destructive[13]. However, both methods can only be used in vitro. The roots of primary teeth can be absorbed physically or pathologically, and it is difficult to obtain complete primary teeth for study in vitro. Cone-beam computed tomography (CBCT) has a higher resolution and can better reflect the 3D shape of the root canal than two-dimensional radiographs[14]. The radiation dose of $\mathrm{CBCT}$ is lower than that of micro-CT, and CBCT has been used for research on permanent teeth in vivo[15]. Therefore, a large number of in vivo normal tooth samples can be obtained through CBCT.

Studies on age-related changes in the root canal morphology of primary molars are indispensable to deeply understand the physiological structure of deciduous teeth and provide a reference for related clinical work. To study age-related changes, observation indices should be determined first. In this study, we aimed to elucidate age-related changes in the root canal morphology of primary molars and determine new indices to detail these changes in root canal anatomy through CBCT.

\section{Materials And Methods}

This study was approved by the Ethics Committee of Peking University School and Hospital of Stomatology (approval number: PKUSSIRB-201949122).

\section{Data collection}

Data of CBCT scans that were originally conducted from April 2018 to November 2019 for the diagnosis or treatment of supernumerary teeth, trauma, cysts, etc., unrelated to this study were retrieved from the Department of Radiology at Peking University Hospital of Stomatology. Radiographic images of systemically healthy children aged 3-8 years were selected. The maxillary first primary molars on these CBCT scans were evaluated as the study objects, and those meeting the following criteria were excluded:

1. abnormality in the crown or root morphology;

2. caries reaching the superficial or deep layer of dentin or pulpal infection caused by another disease;

3. abrasion reaching to the dentin;

4. history of pulpotomy, pulpectomy or preformed metal crown restoration;

5. immature roots or internal or external root absorption;

6. incomplete image or unsatisfactory image quality.

CBCT images were acquired using a 3D Accuitomo type F17 (Morita, Kyoto, Japan) at 80-90 kV and $5 \mathrm{~mA}$, with a field of view of $6 \mathrm{~cm} * 6 \mathrm{~cm}$. The voxel and slice thicknesses were both $0.125 \mathrm{~mm}$. All CBCT exposures were performed by a licenced radiologist strictly following the manufacturer's recommended protocol. The CBCT scans selected were divided into 6 groups by age.

A preliminary study was carried out to estimate the sample size for each age group. The sample size was calculated using PASS 11.0 software, with an alpha error of 0.05 and a known standard deviation of the 
root canal cross-sectional area. The estimated sample size for each age group was 10 teeth.

\section{Index definition and measurement}

The CBCT data were saved in DICOM (Digital Imaging and Communications in Medicine) format and transferred to Mimics 17.0 (Materialise Technologies, Leuven, Belgium). Through preliminary exploration and analysis, the following points and plane were defined to aid with the measurement of each root and canal (Fig. 1a).

Point A: the lowest point of the outer surface of the root furcation along the z-axis.

Point B: the anatomical root tip.

Point C: the point where the canal lumen disappeared towards the root tip for the first time.

Plane D: the plane $1 / 3^{*} L_{R}$ from point A towards the root tip along the z-axis; this plane is perpendicular to the z-axis.

$L_{R}$ : the length of the root measured from point $A$ to $B$ along the z-axis.

$\mathrm{L}_{\mathrm{C}}$ : the length of the canal measured from point $A$ to $\mathrm{C}$ along the $\mathrm{z}$-axis.

If the root canal lumen reappeared after disappearing towards the root tip, it was recorded as a discontinuous canal, as shown in Fig. 1a. The $L_{C}$ and $L_{R}$ were calculated.

The canal segment with a thickness of $0.25 \mathrm{~mm}$ extending from plane $\mathrm{D}$ towards the root tip was taken as the region of interest for 3D reconstruction. First, an appropriate threshold to segment the canal and dentin was selected. The threshold was determined as follows. The threshold range of the lip on the CBCT scan at three different positions was measured, and the difference between the minimum and maximum thresholds of the three positions was calculated. Then, the mean of the three differences was calculated and recorded as " $d$ ". The minimum threshold of the canal image was recorded as "c", and d plus $c$ was defined as " $p$ ", which was recorded as the reference threshold of the canal. Then, the reference threshold " $p$ " was slightly adjusted to improve the accuracy of segmentation according to the operator's judgement.

After determination of a proper canal threshold, a 3D model of the root canal segment of interest was reconstructed using Mimics 17.0 software (Fig. 1b) and measured using 3-Matic 9.0 software (Materialise Technologies, Leuven, Belgium). The detailed procedure is shown in Fig. 2.

The following target measure variables were calculated and recorded:

1) The area of the canal cross-section on plane D, measured using ImageJ (National Institutes of Health, Bethesda, USA).

2) $L_{C} / L_{R}$ : the ratio of the canal length and root length. 
3) $R_{\cdot 15}$ : the ratio of the inner lateral canal wall area with a root canal diameter less than $0.15 \mathrm{~mm}$ and the total inner lateral canal wall area of the segment of interest.

4) $\mathrm{N}_{\text {dis: }}$ : the number of discontinuous root canals.

Before starting the study, 69 canal cross-sections not included in this study were used to assess the consistency of image segmentation with the mean intersection over union (MloU) as an index. The MloU was 0.81 . The intragroup correlation coefficient (ICC) was used to test the consistency of the selection of the defined points in the image. The interexaminer and intraexaminer ICCs were 0.998 and 1, respectively.

\section{Statistical analysis}

Statistical analyses were conducted using SPSS version 20.0 (SPSS, Inc., Chicago, IL, USA). The normality of data obtained from CBCT scans was tested using the Kolmogorov-Smirnov one-sample test. Area and length variables that did not exhibit a normal distribution were analysed using a nonparametric test (Kruskal-Wallis test). The relationships of $\mathrm{R}_{\cdot 15}, \mathrm{~L}_{\mathrm{C}} / \mathrm{L}_{\mathrm{R}^{\prime}}$ and canal cross-sectional area on plane $\mathrm{D}$ with age were analysed using correlation analysis, and the linear regression coefficient between $N_{\text {dis }}$ and age was calculated by binary logistic regression. The significance level was set at $P<0.05$.

\section{Results}

In total, 58 children (14 girls and 44 boys) were enrolled, including 107 maxillary first primary molars. The average age was 6.0 years, ranging from 3.0 to 8.7 years. All teeth were divided into six groups according to age, and the distribution of the teeth across these groups is shown in Table 1.

Table 1

Distribution of maxillary first primary molars across different age groups

\begin{tabular}{|lllllll|}
\hline Age groups (year) & $3 \sim$ & $4 \sim$ & $5 \sim$ & $6 \sim$ & $7 \sim$ & $8 \sim ~$ \\
\hline Teeth $(\mathrm{N})$ & 11 & 22 & 17 & 20 & 18 & 19 \\
\hline
\end{tabular}

The cross-sectional area of each maxillary first primary molar root canal on plane $\mathrm{D}$ is shown in Table 2 . The cross-sectional area of the root canals of mesiobuccal (MB), distobuccal (DB), and palatal (P) roots on plane $\mathrm{D}$ was negatively correlated with age, suggesting that the root canal cross-sectional area decreased with age. Axial CBCT images of teeth from children of different ages (Fig. 3) showed shrinkage of the lumen of all kinds of roots.

In addition, the cross-sectional area of $\mathrm{P}$ root canals was larger than that of $\mathrm{DB}$ and $\mathrm{MB}$ root canals $(P<$ $0.001)$; the cross-sectional area of DB root canals was significantly smaller than that of MB root canals $(P$ $=0.031$ ). 
Table 2

Cross-sectional area on plane $\mathrm{D}$ of all root canals

\begin{tabular}{|c|c|c|c|c|c|c|c|}
\hline \multirow{2}{*}{$\begin{array}{l}\text { Root } \\
\text { canal }\end{array}$} & \multirow{2}{*}{$\begin{array}{l}\text { Age } \\
\text { (year) }\end{array}$} & \multicolumn{4}{|c|}{ Cross-sectional area of root canal $\left(\mathrm{mm}^{2}\right)$} & \multirow[t]{2}{*}{$r_{s}$} & \multirow[t]{2}{*}{$P$} \\
\hline & & Minimum & Maximum & Median & Mean & & \\
\hline \multirow[t]{6}{*}{ MB } & 3 & 0.05 & 0.73 & 0.50 & 0.38 & -0.41 & $<0.001$ \\
\hline & 4 & 0.06 & 0.42 & 0.20 & 0.20 & & \\
\hline & 5 & 0 & 0.31 & 0.14 & 0.14 & & \\
\hline & 6 & 0 & 0.58 & 0.16 & 0.18 & & \\
\hline & 7 & 0 & 0.30 & 0.02 & 0.08 & & \\
\hline & 8 & 0 & 0.47 & 0.11 & 0.12 & & \\
\hline \multirow[t]{6}{*}{ DB } & 3 & 0 & 0.61 & 0.14 & 0.24 & -0.26 & 0.008 \\
\hline & 4 & 0 & 0.30 & 0.13 & 0.11 & & \\
\hline & 5 & 0 & 0.36 & 0.06 & 0.09 & & \\
\hline & 6 & 0 & 0.34 & 0 & 0.08 & & \\
\hline & 7 & 0 & 0.30 & 0.03 & 0.10 & & \\
\hline & 8 & 0 & 0.20 & 0 & 0.06 & & \\
\hline \multirow[t]{6}{*}{$P$} & 3 & 0.28 & 1.69 & 0.75 & 0.85 & -0.42 & $<0.001$ \\
\hline & 4 & 0.34 & 1.11 & 0.70 & 0.67 & & \\
\hline & 5 & 0.20 & 0.95 & 0.66 & 0.63 & & \\
\hline & 6 & 0.22 & 1.38 & 0.59 & 0.58 & & \\
\hline & 7 & 0.06 & 0.73 & 0.44 & 0.42 & & \\
\hline & 8 & 0.06 & 1.16 & 0.42 & 0.47 & & \\
\hline
\end{tabular}

The root length and canal length were calculated and are shown in Table 3 . The length of $P$ roots was greater than $\mathrm{DB}$ roots and less than $\mathrm{MB}$ roots $(P<0.001)$. The length of $\mathrm{MB}, \mathrm{DB}$, and $\mathrm{P}$ root canals was negatively correlated with age, with a correlation coefficient of $-0.55,-0.49$, and -0.49 , respectively $(P<$ 0.001 ), suggesting a decrease in canal length with age. Meanwhile, the $M B, D B$, and $P L_{C} / L_{R}$ was also negatively correlated with age (Table 4, Fig. 4). 
Table 3

Root length and canal length at different ages

\begin{tabular}{|c|c|c|c|c|c|c|c|c|c|c|c|}
\hline \multirow{2}{*}{$\begin{array}{l}\text { Root } \\
\text { canal }\end{array}$} & \multirow{2}{*}{$\begin{array}{l}\text { Age } \\
\text { (year) }\end{array}$} & \multicolumn{4}{|c|}{ Root length } & \multicolumn{6}{|c|}{ Canal length } \\
\hline & & Min & Max & Median & Mean & Min & Max & Median & Mean & $r_{s}$ & $P$ \\
\hline \multirow[t]{6}{*}{ MB } & 3 & 4.75 & 8.50 & 7.75 & 7.09 & 3.0 & 6.0 & 4.88 & 4.45 & -0.55 & 0.000 \\
\hline & 4 & 5.63 & 8.25 & 7.00 & 6.94 & 3.25 & 5.38 & 4.31 & 4.35 & & \\
\hline & 5 & 4.25 & 9.38 & 6.75 & 6.84 & 0.88 & 4.37 & 3.38 & 3.24 & & \\
\hline & 6 & 4.13 & 7.88 & 6.37 & 6.14 & 1.25 & 4.63 & 3.38 & 3.21 & & \\
\hline & 7 & 3.38 & 9.50 & 7.12 & 6.50 & 0.25 & 5.50 & 2.88 & 2.88 & & \\
\hline & 8 & 3.87 & 8.00 & 6.25 & 5.93 & 0 & 5.25 & 2.38 & 2.31 & & \\
\hline \multirow[t]{6}{*}{ DB } & 3 & 4.00 & 6.00 & 4.88 & 4.88 & 1.75 & 4.50 & 2.75 & 3.01 & -0.49 & 0.000 \\
\hline & 4 & 3.25 & 6.25 & 4.57 & 4.65 & 0.25 & 4.37 & 2.57 & 2.31 & & \\
\hline & 5 & 2.25 & 8.50 & 4.50 & 4.77 & 0 & 3.63 & 1.75 & 1.88 & & \\
\hline & 6 & 1.13 & 7.50 & 4.69 & 4.32 & 0 & 3.75 & 1.38 & 1.56 & & \\
\hline & 7 & 2.13 & 5.50 & 3.63 & 3.59 & 0 & 3.75 & 1.06 & 1.27 & & \\
\hline & 8 & 2.38 & 6.88 & 4.13 & 4.29 & 0 & 3.25 & 0.75 & 1.05 & & \\
\hline \multirow[t]{6}{*}{$P$} & 3 & 4.12 & 8.50 & 6.38 & 6.24 & 3.75 & 6.87 & 4.75 & 5.02 & -0.49 & 0.000 \\
\hline & 4 & 3.88 & 9.25 & 5.50 & 5.89 & 2.50 & 7.12 & 4.63 & 4.68 & & \\
\hline & 5 & 3.13 & 6.88 & 5.63 & 5.30 & 2.88 & 5.50 & 3.87 & 3.96 & & \\
\hline & 6 & 3.00 & 7.62 & 5.38 & 5.39 & 2.63 & 6.25 & 3.94 & 4.06 & & \\
\hline & 7 & 3.25 & 7.12 & 5.38 & 5.13 & 2.50 & 4.87 & 3.25 & 3.46 & & \\
\hline & 8 & 2.50 & 8.25 & 4.63 & 4.99 & 2.12 & 5.50 & 3.50 & 3.50 & & \\
\hline
\end{tabular}

Statistical analysis showed a positive correlation between the MB R.15 and age $\left(r_{s}=0.39, P<0.001\right)$, indicating that the canal diameter decreased with age (Table 4). No similar results was obtained for DB and $P$ roots. 
Table 4

Correlations of $L_{C} / L_{R}, R_{\cdot 15}$ and age

\begin{tabular}{|c|c|c|c|c|c|c|c|c|c|}
\hline & \multicolumn{6}{|c|}{ Age (year) } & \multirow[t]{2}{*}{$r_{s}$} & \multirow[t]{2}{*}{$P$} \\
\hline & & 3 & 4 & 5 & 6 & 7 & 8 & & \\
\hline \multicolumn{10}{|c|}{$L_{C} / L_{R}$} \\
\hline \multirow[t]{3}{*}{ MB } & & & & & & & & -0.39 & $<0.001$ \\
\hline & Median & 0.65 & 0.64 & 0.48 & 0.56 & 0.46 & 0.40 & & \\
\hline & Mean & 0.62 & 0.62 & 0.48 & 0.53 & 0.46 & 0.39 & & \\
\hline \multirow[t]{3}{*}{ DB } & & & & & & & & -0.42 & $<0.001$ \\
\hline & Median & 0.56 & 0.50 & 0.36 & 0.33 & 0.30 & 0.18 & & \\
\hline & Mean & 0.62 & 0.48 & 0.40 & 0.33 & 0.36 & 0.24 & & \\
\hline \multirow[t]{3}{*}{$P$} & & & & & & & & -0.29 & 0.002 \\
\hline & Median & 0.83 & 0.79 & 0.79 & 0.83 & 0.70 & 0.77 & & \\
\hline & Mean & 0.81 & 0.80 & 0.76 & 0.77 & 0.69 & 0.72 & & \\
\hline \multicolumn{10}{|l|}{$\mathrm{R}_{.15}$} \\
\hline \multirow[t]{3}{*}{ MB } & & & & & & & & 0.39 & $<0.001$ \\
\hline & Median & 0 & 0 & 0.18 & 0.29 & 1 & 0.57 & & \\
\hline & Mean & 0.08 & 0.07 & 0.31 & 0.41 & 0.57 & 0.51 & & \\
\hline \multirow[t]{3}{*}{ DB } & & & & & & & & 0.16 & 0.098 \\
\hline & Median & 0.68 & 0.99 & 0.89 & 1 & 1 & 1 & & \\
\hline & Mean & 0.48 & 0.48 & 0.65 & 0.65 & 0.61 & 0.66 & & \\
\hline \multirow[t]{3}{*}{$P$} & & & & & & & & 0.09 & 0.349 \\
\hline & Median & 0 & 0 & 0 & 0 & 0 & 0 & & \\
\hline & Mean & 0.01 & 0 & 0 & 0.02 & 0.07 & 0.02 & & \\
\hline
\end{tabular}

The number of discontinuous root canals in different age groups was counted, and the percentage of discontinuous canals was calculated. The percentage of discontinuous MB, DB, and P root canals increased with age (Fig. 5). Binary logistic regression analysis revealed that the risk of discontinuous MB, $P$ and DB roots canals significantly increased with age (Table 5). 
Table 5

Results of binary logistic analysis for $\mathrm{N}_{\text {dis }}$ and age

\begin{tabular}{|cllllll|}
\hline Variable & $B$ & $P$-value & OR & \multicolumn{2}{c|}{$95 \%$ Cl for OR } \\
\cline { 4 - 7 } & & & & & Lower & Upper \\
\hline$N_{\text {dis }}$ & MB & 0.48 & 0.001 & 1.61 & 1.20 & 2.15 \\
\cline { 2 - 7 } & DB & 0.39 & 0.003 & 1.48 & 1.14 & 1.91 \\
\hline & $P$ & 0.47 & 0.043 & 1.60 & 1.02 & 2.51 \\
\hline
\end{tabular}

$\mathrm{N}_{\text {dis }}$, number of discontinuous root canals; $\mathrm{B}$, unstandardized coefficient; $\mathrm{Cl}$, confidence interval; DB, distobuccal root; $\mathrm{MB}$, mesiobuccal root; $\mathrm{OR}$, odds ratio; $\mathrm{P}$, palatal root.

\section{Discussion}

It is difficult to obtain complete primary teeth because of the physiological or pathological absorption of deciduous roots. CBCT provides a feasible method by which to study the root canal morphology of deciduous teeth. In this study, we obtained complete 3D structural data of deciduous teeth through CBCT. Different from previous micro-CT or nano-computed tomography (nano-CT) in vitro studies[13, 16], the teeth included in this study were all normal teeth or teeth with caries not exceeding the superficial dentin, with healthy pulp-dentin complexes in vivo. Such samples can reflect the morphology of the teeth under the physiological state and are of great significance for obtaining thorough knowledge of the normal anatomy of primary teeth.

Indices for root canal ageing should not only reflect changes in the root canal morphology on CBCT but also have clinical significance to serve as a reference for related treatment. The canal diameter is directly related to the file size [17]. Because of the irregular cross-sectional shape of the canal, measuring only the maximum and minimum diameter of the root canal cannot clarify the relationship between the inner diameter of the root canal and the file. In this study, the diameter of the canal segment of interest was measured at all positions, and the proportion of the root canal with a diameter less than $0.15 \mathrm{~mm}$, i.e., the $\mathrm{R}_{\text {.15 }}$, was calculated. The cut-off value of $0.15 \mathrm{~mm}$ was chosen because the $\# 15 \mathrm{~K}$-file is commonly used as the initial manual file to prepare the root canals of deciduous molars. This observation variable can reflect the possibility of the file entering the canal and the proportion of the lateral wall area of the region of interest that can be touched by the \#15 K-file on plane $\mathrm{D}$. This measurement index can provide more information than the diameter of the root canal in a certain direction. The cross-sectional area of the canal can directly show changes in the canal without being affected by the cross-sectional canal shape, which is a common index used in root canal measurement[18, 19].

In this study, the variable $\mathrm{R}_{\cdot 15}$ of $\mathrm{MB}$ roots increased with age, and the cross-sectional area of MB root canals on plane $D$ decreased with age, indicating a decrease in the canal diameter. Similar results were found in the orifice of the mesial root canals of mandibular primary molars $(P<0.01)$ [8]. These findings indicate that as children age, the canal portion with an inner diameter larger than $0.15 \mathrm{~mm}$ decreases, and 
the \#15 K-file becomes less likely to enter the root canal on plane D. Furthermore, we observed that most MB canal cross-sections were oval or flattened on plane D (Fig. 3). The presence of an oval canal and a smaller canal diameter increase the difficulty of mechanical and chemical canal instrumentation, resulting in larger areas of the dentin wall being untouched[20]. However, the $\mathrm{R}_{{ }_{15}}$ of $\mathrm{DB}$ and $\mathrm{P}$ canals was not found to correlate with age in this study. This is because most DB canal diameters were smaller than $0.15 \mathrm{~mm}$ on plane $D$ at these young ages. In contrast to $D B$ canals, for $P$ canals, nearly all diameters on plane $D$ were larger than $0.15 \mathrm{~mm}$ for all ages. However, the area of DB and P canals on plane D decreased significantly with age, reflecting age-related changes in the canals.

Changes in the anatomy of the lower half of the root canal were reflected by the variables $L_{C} / L_{R}$ and $N_{\text {dis. }}$. The results showed that $L_{C} / L_{R}$ decreased and $N_{\text {dis }}$ increased significantly with advancing age. Secondary dentin deposition causes root canal shrinkage, which can even block small-diameter canals, resulting in canal shortening. However, canal disappearance was usually observed to be incomplete on CBCT, and in some canals, the minor lumen could still be observed under the deposited dentin. This may be caused by different rates of dentin deposition and small differences in the inner diameter of the root canal. Because of the lower resolution, these discontinuous canals may show no root lumen on two-dimensional clinical radiographies, which may affect the accuracy of root canal working length confirmation and both lead to incomplete root canal preparation and increase the risk of leaving residual infectious substances.

Because the lengths of the three roots of the maxillary first deciduous molars were significantly different, we chose to divide the roots into three equal parts to observe the changes in the canals with age, as in previous studies[18]. During the experiment, it was found that for children over 5 years old, most of the DB root canal lumen and the majority of the MB root canal lumen could not be seen on the plane positioned at the midpoint of the apical two-thirds of the root length. Therefore, the plane where the midpoint of the coronal two-thirds of the root length lies was chosen as the measurement plane, which was named plane D.

In this study, four feasible observation indices were successfully developed for careful observation of the root canal morphology of deciduous teeth. It is hoped that more clinicians will participate in relevant work in the future to revise and improve these indices so that they can more accurately reflect the morphological characteristics of the root canal morphology of deciduous teeth and improve our understanding of the root canal morphology of deciduous teeth.

There are some limitations to this study. First, for the study of age-related changes, obtaining CBCT scans of the same child at different ages is the best approach. However, children are sensitive to radiation, so CBCT cannot be performed only for scientific research. Therefore, it is more practical to choose scans from children of different age groups for comparison. Second, due to the strict indications for CBCT in young children, although the teeth included met the sample size requirements, all children were from one clinical centre, and the results of some indices may be affected by individual differences. However, the changes with age were still obvious. The final research results need to be verified by studies with larger samples at multiple clinical centres. Third, due to the limitations of the resolution of $\mathrm{CBCT}$, only the main root canal could be studied, and root canals smaller than the voxel size could not be identified. 


\section{Conclusions}

New indices were successfully developed in this study to demonstrate age-related changes in the root canals of primary molars by CBCT.

\section{Abbreviations}

CBCT

cone-beam computed tomography; DB:distobuccal; MB:mesiobuccal; P:palatal; 3D:three-dimensional; MloU:mean intersection over union;RCT:root canal treatment; STL:stereo lithography; micro-CT:microcomputed tomography; DICOM:digital imaging and communications in medicine; ICC:intragroup correlation coefficient; nano-CT:nano-computed tomography.

\section{Declarations}

\section{Ethics approval and consent to participate}

This study was conducted in accordance with the tenets of the Declaration of Helsinki for research involving human subjects, and approved by the Ethics Committee of Peking University School and Hospital of Stomatology (approval number: PKUSSIRB-201949122).

\section{Consent for publication}

Not applicable.

\section{Availability of data and materials}

The datasets used and analysed during the current study are available from the corresponding author on reasonable request.

\section{Competing interests}

The authors declare that they have no competing interests.

\section{Funding}

This project was supported by grant Capital's Funds for Health Improvement and Research (Grant number: 2020-2-4105).

\section{Authors' contributions}

Bin Xia and Guili Dou have made substantial contributions to all the process of the study. Guili Dou wrote the manuscript text, and Bin Xia revised it. Zhipeng Sun has made contributions to the data collection, and Yijiao Zhao has made contributions to the date analysis. All authors have read and approved this manuscript. 


\section{Acknowledgements}

The authors thank Xueying Li, from Peking University First Hospital, for help with the statistical analyses and the American Journal Experts editing team for their language editing services.

\section{References}

1. Chen Y, Li H, Li M, Yang L, Sun Q, Chen K. Analysis of survival and factors associated with failure of primary tooth pulpectomies performed under general anaesthesia in children from South China. Int $\mathrm{J}$ Paediatr Dent. 2019;30(2):225-33.

2. Atieh MA. Root and canal morphology of maxillary first premolars in a Saudi population. $\mathrm{J}$ Contemp Dent Pract. 2008;9(1):46-53.

3. Fan W, Fan B, Gutmann JL, Fan M. Identification of a C-shaped Canal System in Mandibular Second Molars-Part III: Anatomic Features Revealed by Digital Subtraction Radiography. J Endod. 2008;34(10):1187-90.

4. Weng X-L, Yu S-B, Zhao S-L, Wang H-G, Mu T, Tang R-Y, Zhou X-D. Root Canal Morphology of Permanent Maxillary Teeth in the Han Nationality in Chinese Guanzhong Area: A New Modified Root Canal Staining Technique. J Endod. 2009;35(5):651-6.

5. Philippas GG. Influence of Occlusal Wear and Age on Formation of Dentin and Size of Pulp Chamber. J Dent Res. 1961;40(6):1186-98.

6. Zheng QH, Wang Y, Zhou XD, Wang Q, Zheng GN, Huang DM. A cone-beam computed tomography study of maxillary first permanent molar root and canal morphology in a Chinese population. $J$ Endod. 2010;36(9):1480-4.

7. Martins JNR, Ordinola-Zapata R, Marques D, Francisco H, Caramês J. Differences in root canal system configuration in human permanent teeth within different age groups. Int Endod J. 2018;51(8):931-41.

8. Ge L, Zheng S, Zhang T, Jiu B, Zhang Y, Hou T. Morphologic study of the root canal orifices in human mandibular primary molars. J Modern Stomatol. 2002;16(4):363-5.

9. Amano M, Agematsu H, Abe S, Usami A, Matsunaga S, Suto K, Ide Y. Three-dimensional analysis of pulp chambers in maxillary second deciduous molars. J Dent Child. 2006;34(7):503-8.

10. Hibbard ED, Ireland RL. Morphology of the root canals of the primary molar teeth. J Dent Child. 1957;24:250-7.

11. Vertucci FJ. Root canal morphology of mandibular premolars. J Am Dent Assoc. 1978;97(1):47-50.

12. Neelakantan PM, Subbarao CB, Subbarao CVM. Comparative Evaluation of Modified Canal Staining and Clearing Technique, Cone-Beam Computed Tomography, Peripheral Quantitative Computed Tomography, Spiral Computed Tomography, and Plain and Contrast Medium-enhanced Digital Radiography in Studying Root Canal Morphology. J Endod. 2010;36(9):1547-51.

13. Lee JK, Yoo YJ, Perinpanayagam H, Ha BH, Lim SM, Oh SR, Gu Y et al. Three-dimensional modelling and concurrent measurements of root anatomy in mandibular first molar mesial roots using microcomputed tomography. Int Endod J. 2015;48(4):380-9. 
14. Li Y-h, Bao S-j, Yang X-w, Tian X-m, Wei B, Zheng Y-I. Symmetry of root anatomy and root canal morphology in maxillary premolars analyzed using cone-beam computed tomography. Arch Oral Biol. 2018;94:84-92.

15. Martins JNR, Marques D, Silva EJNL, Caramês J, Versiani MA. Prevalence Studies on Root Canal Anatomy Using Cone-beam Computed Tomographic Imaging: A Systematic Review. J Endod. 2019;45(4):372 - 86.e4.

16. Torres-Ramos G, Lucisano MP, Blanco-Victorio DJ, Ramírez Sotelo LR, Nelson Filho P, Silva RAB, Silva LAB. Root canal conicity estimation of primary maxillary central and lateral incisors-A study by NanoCT. Int J Paediatr Dent. 2020;30(6):764-74.

17. Peters $O A$, Laib A, Ruegsegger $P, B a r b a k o w ~ F$. Three-dimensional analysis of root canal geometry by high-resolution computed tomography. J Dent Res. 2000;79(6):1405-9.

18. Kurthukoti AJ, Sharma P, Swamy DF, Shashidara R, Swamy EB. Computed Tomographic Morphometry of the Internal Anatomy of Mandibular Second Primary Molars. Int J Clin Pediatr Dent. 2015;8:202-7.

19. Fumes AC, Sousa-Neto MD, Leoni GB, Versiani MA, Da Silva LAB, Da Silva RAB, Consolaro A. Root canal morphology of primary molars: a micro-computed tomography study. European Archives of Paediatric Dentistry. 2014;5(15):317-26.

20. Paque F, Balmer M, Attin T, OA P. Preparation of oval-shaped root canals in mandibular molars using nickel-titanium rotary instruments: a micro-computed tomography study. J Endod. 2010;36(4):703-7.

\section{Figures}



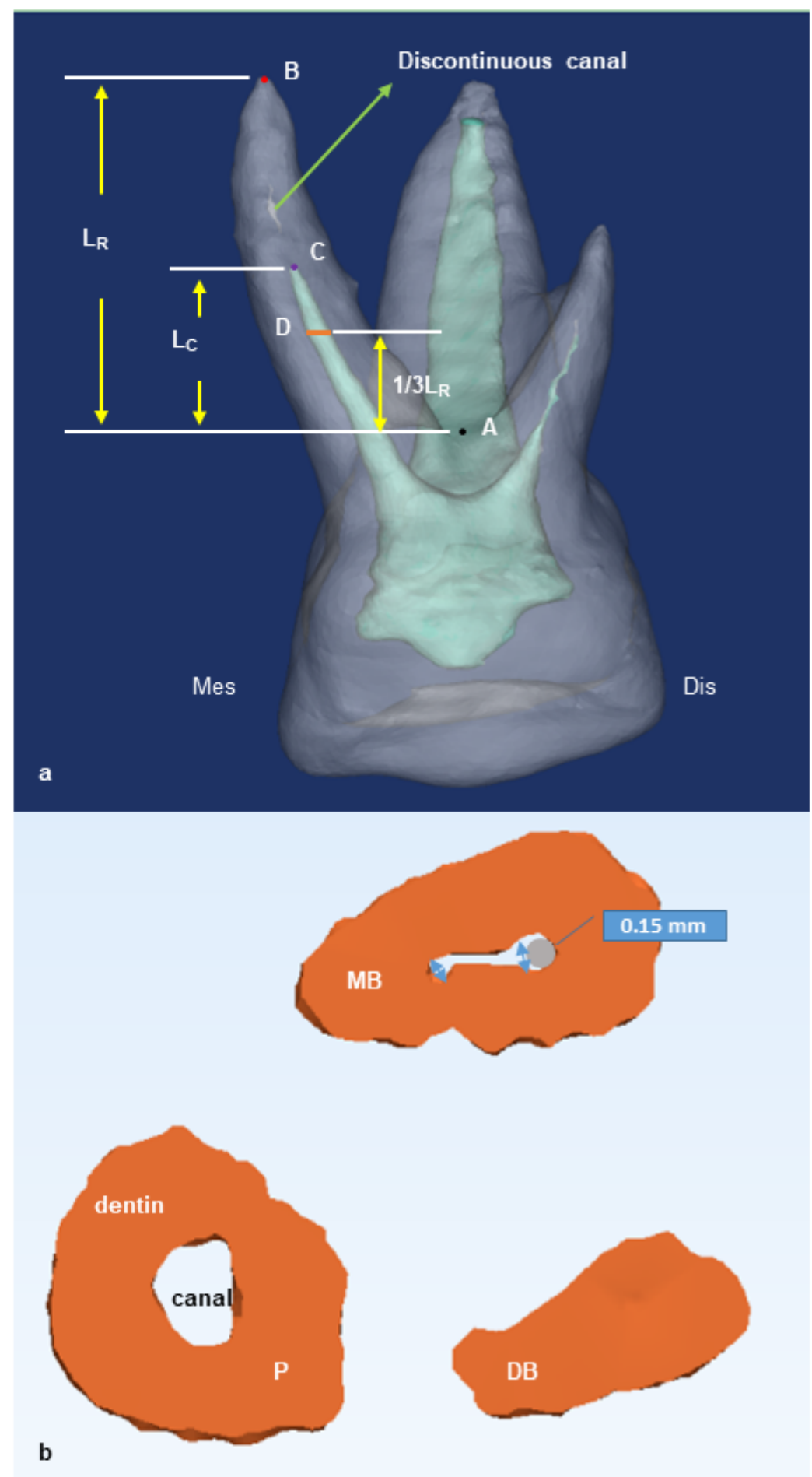

\section{Figure 1}

Representative 3D reconstruction of a maxillary first primary molar. a: 3D reconstruction model of a maxillary first primary molar. Points $A, B$, and $C$, plane $D$, and $L C$ and $L R$ are as indicated. b: Root canal segments with a thickness of $0.25 \mathrm{~mm}$ starting from plane $D$ towards root tip taken as the region of interest of $M B, D B$, and $P$ roots and reconstructed with Mimics 17.0 software. The grey circle represents the tip of the \#15 K-file, and the blue arrows represent the root canal diameter at different positions. A, the lowest 
point of the outer surface of the root furcation; $B$, the anatomical root tip; $C$, the point where the canal disappears towards the root tip for the first time; DB, distobuccal; Dis, distal surface; LC, length of the canal measured from point $A$ to $C$ along the tooth axis; $L R$, length of the root measured from point $A$ to $B$ along the tooth axis; $M B$, mesiobuccal; Mes, mesial surface; $P$, palatal; Plane $D$, the plane $1 / 3^{*} L R$ from point $A$ along the tooth axis.
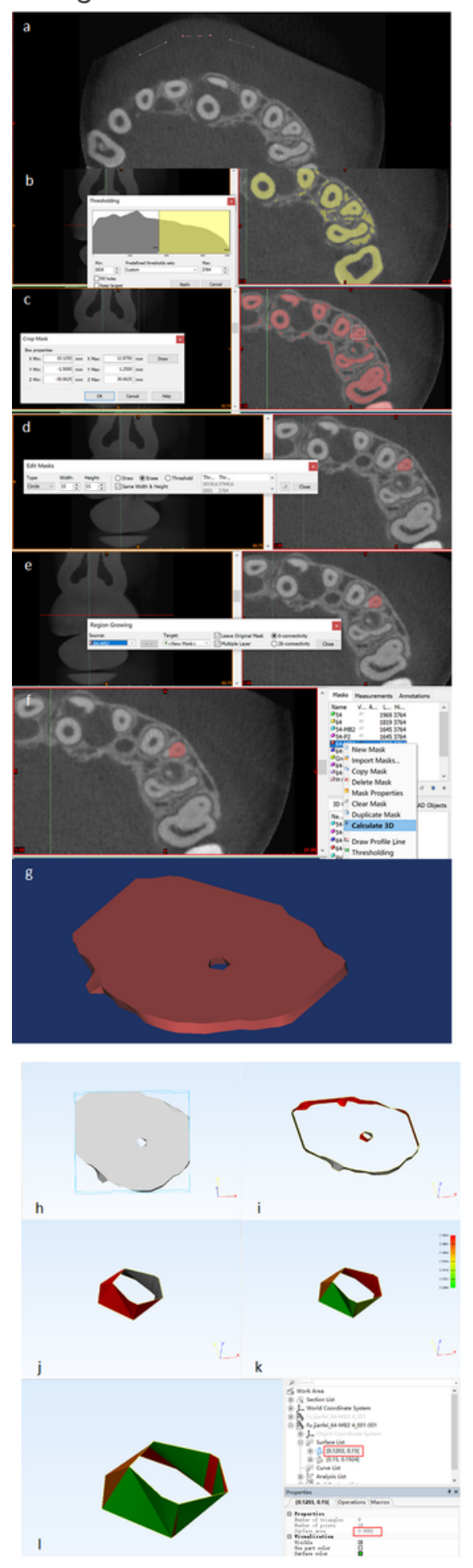

Figure 2 
Software operation flowchart for 3D reconstruction and root canal diameter measurement in the region of interest. a: Measurement of the threshold range of the lip in the CBCT scans at three different positions. b: Selection of the proper threshold to segment the canal and dentin. c-e: Selection of the region of interest from the CBCT image. f-g: Reconstruction of the root canal of the target area to obtain a segmented 3D reconstruction of the root canal. h-j: Extraction of the inner surface of the 3D root canal model. k: Measurement of the diameter of the root canal. I: Division of the extracted inner surface of the root canal into two parts according to whether the diameter was less than $0.15 \mathrm{~mm}$, followed by determination of the area (indicated by a red rectangle).
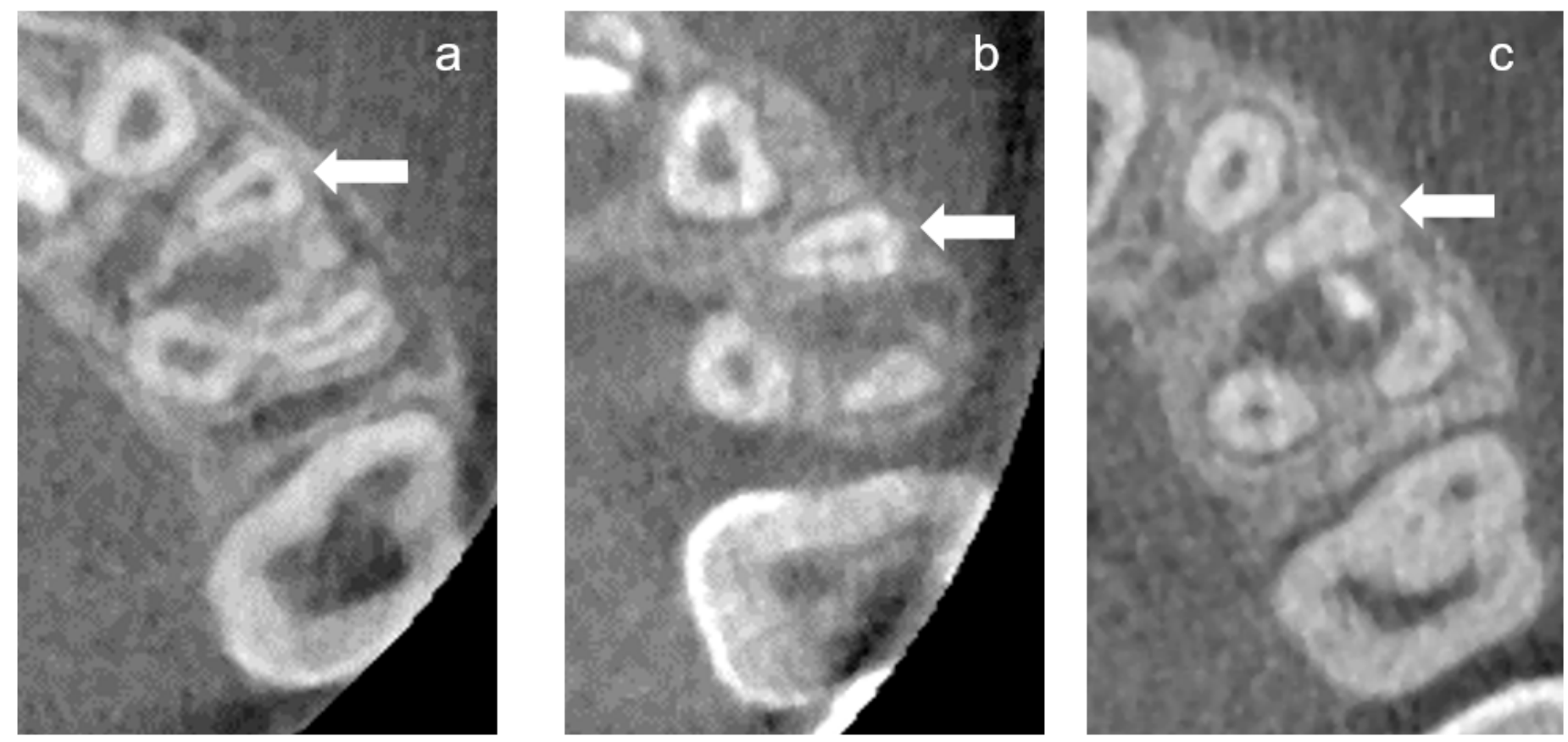

\section{Figure 3}

Decrease in the canal diameter of the left maxillary first primary molar with age. (a) MB canal of a 3-yearold child. (b) MB canal of a 6-year-old child. (c) MB canal of an 8-year-old child. From a to c, the lumen of the MB canal decreases. MB, mesiobuccal.
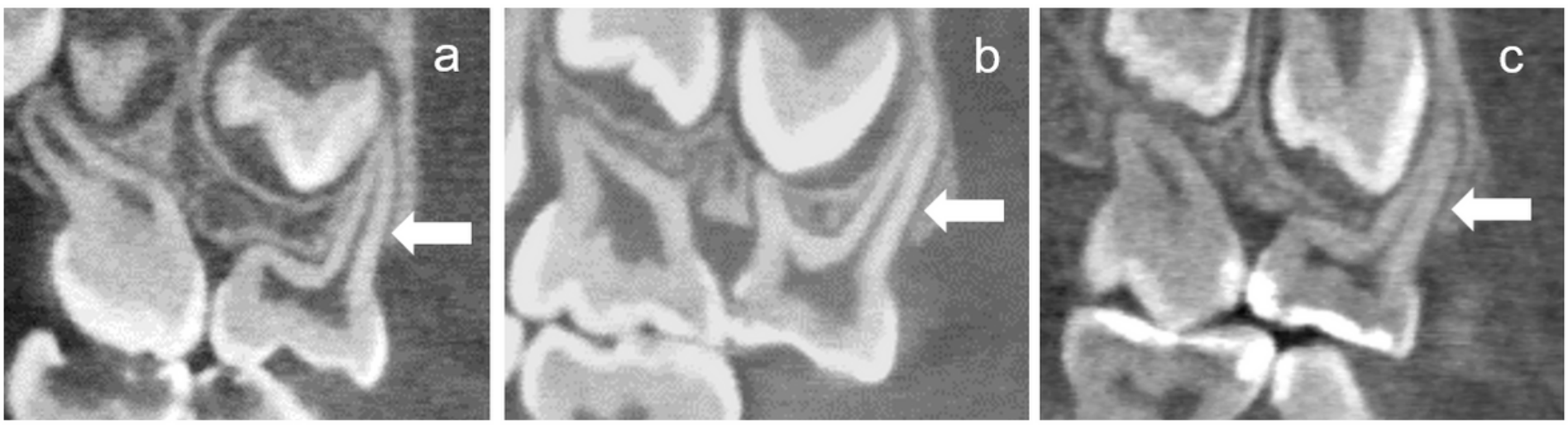

Figure 4 
Decrease in the canal length of the left maxillary first primary molar relative to the root length, with a decrease in the LC/LR. (a) MB canal of a 4-year-old child. (b) MB canal of a 6-year-old child. (c) MB canal of an 8-year-old child. LC, length of the canal along the tooth axis measured from the lowest point of the outer surface of the root furcation to the point where the canal disappeared towards the root tip for the first time; $L R$, length of the root measured along the tooth axis from the lowest point of the outer surface of the root furcation to the anatomical root tip; MB, mesiobuccal.

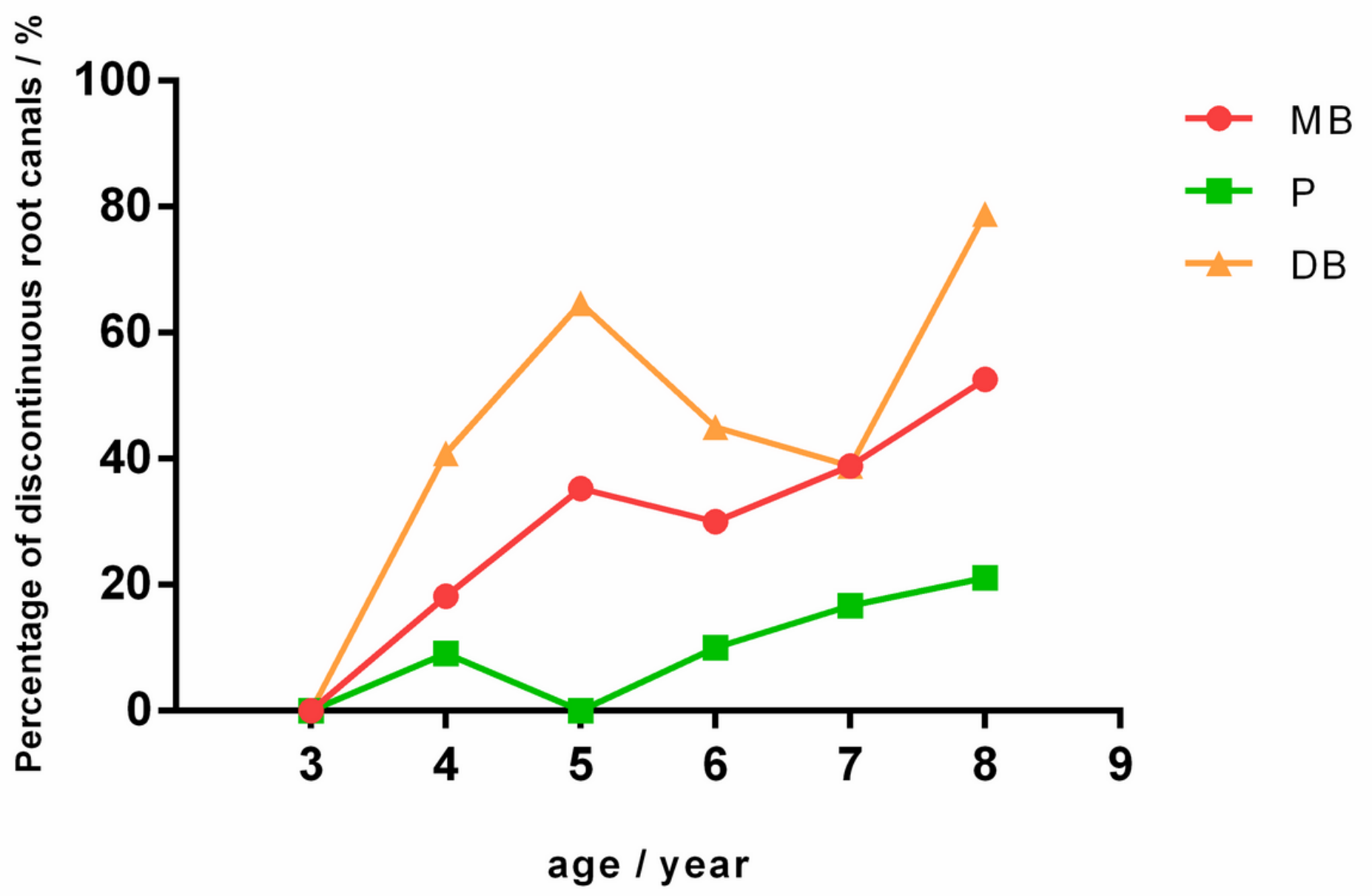

Figure 5

Percentage of discontinuous mesiobuccal, distobuccal and palatal root canals at different ages. 